\title{
Cercle pédagogique et analyse de cas: dispositifs complémentaires de réflexion collective au coeur de la formation initiale des enseignants
}

\section{Mylène Leroux Université du Québec en Outaouais (Canada) \\ Isabelle Vivegnis \\ Université du Québec en Outaouais (Canada)}

\author{
Pedagogical circle and case analysis: Complementary \\ pedagogical approaches to collective reflection \\ in preservice teacher training.
}

$\mathrm{R}$

ésumé

Certains chercheurs considèrent la réflexion comme une métacompétence favorisant le développement des autres compétences professionnelles en enseignement.

II importe ainsi que la formation initiale permette aux personnes étudiantes stagiaires (PES) d'apprendre à réfléchir de manière autonome, c'est-à-dire de s'engager elles-mêmes dans ce processus sans que cela leur soit demandé. C'est dans cette optique que deux dispositifs pédagogiques de réflexion collective ont été adaptés puis expérimentés avec des PES en 2017 : 1) le cercle pédagogique et 2) la démarche d'analyse de cas. Les commentaires des PES révèlent un bilan intéressant quant aux apprentissages perçus, aux apports et à la complémentarité des deux dispositifs, offrant ainsi des pistes pour la formation initiale des enseignants.

Mots-clés

Formation initiale à l'enseignement, réflexion sur la pratique, identité professionnelle, dispositifs pédagogiques, cercles pédagogiques, démarche d'analyse de cas, réflexion collective.

Abstract Some researchers consider teacher reflection a metacompetency that enhances other professional teaching competencies. As such, teacher education should enable student teachers to reflect independently, that is, to engage in reflection without being asked to do so. In 2017, two pedagogical approaches to collective reflection were adapted and became the subject of an experiment with student teachers: 1 ) the pedagogical circle and 2) case analysis. The student teachers' comments revealed interesting results regarding perceived learning as well as the contributions and complementarity of the two approaches - insights that may be useful in improving preservice teacher training.

Keywords

Teacher education, teacher reflection, professional identity, pedagogical approaches, pedagogical circle, case analysis, collective reflection.

\section{Introduction}

$\mathrm{Au}$ Québec, la réflexion est une composante importante de la compétence à s'engager dans une démarche individuelle et collective de développement professionnel chez les enseignants (Leroux, 2010). Cette compétence est jugée nécessaire pour permettre à l'enseignant de s'adapter aux changements et à une réalité toujours nouvelle du milieu professionnel et social que représente l'école, de même qu'à l'évolution de la profession (Ministère de l'Éducation du Québec [MEQ], 2001). Certains chercheurs soutiennent même que la réflexion est une métacompétence qui favorise le développement des autres compétences professionnelles (Correa Molina, Collin, Chaubet et Gervais, 2010). Selon Chaubet (2010), la réflexion permet en quelque sorte d'amener des « mouvements pollinisateurs " (p. 60) entre la pratique, l'expérience et la théorie en favorisant une intégration féconde de ces éléments chez l'apprenant. Il importe ainsi que la formation initiale permette aux personnes étudiantes stagiaires (PES) d'apprendre à réfléchir de manière autonome pour apprendre à enseigner (Svojanovsky, 2017), c'est-à-dire à s'engager de leur propre gré dans ce processus, puis à tirer profit de leur stage, car « l'expérience sur le tas ne suffit plus, elle prend du sens et sera professionnalisante si elle est explicitement mise en mental, en réflexion » (Donnay, 2008, p.XI).

Par ailleurs, la contribution de la dimension collective de la réflexion est souvent mise de l'avant comme étant une composante importante (Collier, 2010; Correa Molina et al., 2010). En effet, le dialogue permettrait aux stagiaires d'élargir leur compréhension des autres et d'eux-mêmes. Or, la culture du travail collectif est peu intégrée dans le milieu professionnel (Thibodeau, Dussault, Frenette et Royer, 2011). Selon Portelance et Durant (2006), il faudrait amorcer le changement de pratiques dès la formation initiale. Par un processus de 
questionnement collectif suscitant la verbalisation et la justification d'idées, la compréhension que les PES ont des autres et d'elles-mêmes serait davantage explicitée, ce qui favoriserait une compréhension améliorée des situations.

Ce sont les raisons pour lesquelles notre intention pédagogique fut d'adapter puis d'expérimenter deux dispositifs de réflexion collective auprès de PES en formation à l'enseignement : 1) le cercle pédagogique et 2) la démarche d'analyse de cas.

\section{Contexte}

L'étude s'est déroulée dans le cadre de deux cours universitaires faisant partie du programme de formation au baccalauréat en éducation préscolaire et en enseignement primaire, et ce, sur une amplitude de deux trimestres. Le premier cours, le Laboratoire d'initiation à la pratique en éducation préscolaire et en enseignement primaire, a lieu en alternance avec le premier stage de formation pratique et se déroule lors du trimestre d'hiver de la première année de formation. Le dispositif pédagogique faisant l'objet de l'investigation dans ce cours, le cercle pédagogique, est une adaptation d'une approche réflexive proposée par Fenstermacher (1987a, 1987b) et adaptée par Correa Molina et Gervais (2010). Concrètement, les PES ont été invitées à capter divers extraits vidéos de leur enseignement en contexte de stage I. Puis, en s'appuyant sur des extraits choisis (révélant une situation satisfaisante ou problématique) et totalisant environ une dizaine de minutes, elles ont participé à des cercles pédagogiques avec deux ou trois de leurs pairs pour expliciter leur pratique et leurs choix pédagogiques. Le rôle des pairs était notamment de soutenir la personne à analyser et expliciter sa pratique en lui posant diverses questions de précision au sujet du déroulement, des élèves, des intentions, des motifs d'action, etc. Les cercles pédagogiques ont duré entre 30 et 45 minutes pour chaque PES participante, incluant le visionnage des extraits par le sous-groupe puis les discussions s'ensuivant. Deux séances de trois heures ont été consacrées en bonne partie à ces cercles pédagogiques et chaque équipe a réalisé trois ou quatre cercles, selon le nombre de participantes. À l'issue de ces cercles pédagogiques, les PES ont fait l'analyse individuelle de leurs arguments pratiques, évoqués lors des discussions, pour en dégager divers apprentissages, prises de conscience et pistes de développement pour la suite de leur formation. Pour ce premier dispositif, ce sont donc des « activités de travail "réelles", avec public authentique " (Chaubet, 2010, p. 71) qui ont été réalisées.

Le second cours, le Séminaire d'intégration II, a lieu en concomitance avec le deuxième stage de formation pratique et se déroule lors du trimestre d'automne subséquent, au début de la deuxième année de formation. Le dispositif pédagogique faisant l'objet de l'investigation dans ce cours, l'analyse de cas, est une adaptation d'une approche réflexive proposée par Korthagen et Kessels (1999) et adaptée par Leroux (2010). Ici, les PES ont été invitées à analyser une situation problématique vécue en stage par une tierce personne. En équipes de 3 ou 4 personnes, les PES devaient sélectionner un cas parmi trois proposés. Un maximum de deux équipes pouvait travailler sur le même cas. En équipe, les PES devaient réaliser une démarche d'analyse du cas en cinq phases en suivant le modèle proposé, le tout sur une durée d'environ 45 minutes. À la phase 1 (5 minutes), les PES devaient parcourir les cas pour en sélectionner un. À la phase 2 (10 minutes), elles devaient tenter d'identifier les faits et les besoins des élèves en découlant. À la phase 3 (10 minutes), elles devaient formuler un problème et en faire ressortir les causes possibles. À la phase 4 (10 minutes), elles devaient proposer des pistes de 
solutions pour résoudre le cas. Enfin, à la phase 5 (10 minutes), elles devaient porter un jugement sur les solutions envisagées en en identifiant les avantages et les inconvénients. À l'issue de l'analyse des cas en équipes, les PES devaient faire un compte-rendu écrit de leur démarche et une courte présentation orale. Pour ce second dispositif, nous nous sommes ainsi servies de « situations d'apprentissage à l'aide de "milieux construits" (Chaubet, 2010, p. 73), des simulations authentiques, mais non vécues par les principaux protagonistes et donc à moindre risque pour eux (Chaubet, 2010).

\section{Objectif du texte}

Considérant qu'assez peu d'études se centrent sur l'analyse des apprentissages des PES au moment de leur formation initiale (Vesterinen, Toom et Krokfors, 2014), le présent texte vise à décrire les retombées de deux dispositifs pédagogiques de réflexion collective dans le cadre de la formation pratique, et ce, selon deux angles d'analyse :

1. les apprentissages perçus par les PES;

2. les apports respectifs des deux dispositifs en vue de dégager leur complémentarité.

\section{Cadre conceptuel}

Les deux dispositifs pédagogiques dont il est ici question reposent sur deux modèles conceptuels de la réflexion, détaillés ci-après. Par ailleurs, puisque les deux dispositifs visaient notamment à stimuler le développement de l'identité professionnelle, nous apportons ensuite quelques clarifications conceptuelles à cet égard.

\section{Réflexion}

Deux modèles conceptuels de réflexion sont à la base des dispositifs ici présentés: d'abord, l'argumentation pratique pour les cercles pédagogiques lors du laboratoire, ainsi que le modèle ALACT pour l'analyse de cas en séminaire.

\section{Premier modèle conceptuel : argumentation pratique}

Une des intentions des cercles pédagogiques était de susciter l'explicitation des fondements de l'agir professionnel chez les apprenants. Cette explicitation permet de mettre au jour ce qui n'est pas observable dans la pratique et de rendre le savoir d'expérience conscient et discursif. Pour ce faire, nous avons retenu une adaptation de l'approche de Fenstermacher (1987a, 1987b) par Correa Molina et Gervais (2010), qui consiste à utiliser la rétroaction vidéo pour amener la PES à expliquer les raisons de son agir professionnel. Cette approche mobilise le concept d' « argument pratique », qui rend possible « une systématisation du processus de réflexion sur la pratique. Il favoriserait également la mise en relation du savoir issu de la recherche et du savoir issu de l'action » (p. 235). L'approche permettrait d'améliorer

la compréhension des actions que pose un enseignant ou une enseignante au quotidien, améliorant ainsi sa formation initiale et continue (Vesterinen et al., 2014). 
Pour élaborer un argument, deux temps sont en principe requis : d'abord l'explicitation, puis la reconstruction. Lors de l'explicitation, il s'agit de formuler des arguments pratiques traduisant les motifs d'agir, le tout reposant sur un dialogue avec un ou plusieurs pairs (appelés Autres), qui fait émerger les fondements de l'action. Fenstermacher (1987a, 1987b) décrit ces arguments sous la forme de quatre différents types de prémisses : 1) les valeurs (buts, finalités, croyances), 2) les savoirs théoriques (justifications théoriques, lectures, cours), 3) l'expérience (confirmation par les faits) et 4) le contexte d'action (contraintes, limites, éléments contextuels). Pour ce qui est de la reconstruction, elle consiste à évaluer la justesse des arguments pratiques formulés dans un premier temps.

À l'instar de Correa Molina et Gervais (2010), nous nous sommes ici centrées sur l'explicitation, en laissant aux PES le choix des extraits vidéos à partir desquels discuter (un moment significatif pour elles). Nous les avons ensuite guidées vers une verbalisation de leurs prémisses, sans les obliger à formuler un argument pratique formel. Relevons par ailleurs deux divergences entre l'approche des chercheurs susmentionnés et la nôtre : 1) dans leur cas, les équipes comportaient à la fois des chercheurs, des stagiaires, de même que leurs formateurs (superviseurs ou enseignants associés) alors que, dans notre cas, les équipes étaient uniquement constituées de PES; 2) les chercheurs ont employé cette approche comme instrument de collecte des données alors que, dans notre cas, elle a d'abord été utilisée comme dispositif pédagogique, dont la mise en œuvre a abouti à un processus de recherche en vue d'en documenter les retombées ${ }^{1}$.

\section{Deuxième modèle conceptuel : ALACT}

En ce qui concerne l'analyse de cas, qui visait à aider les PES à résoudre des situations problématiques, nous avons retenu le modèle ALACT de Korthagen et Kessels (1999), adapté par Leroux (2010). Ce modèle conceptuel de processus de réflexion structurée comprend cinq phases formant une boucle itérative et spiralaire. La phase 1 consiste en la pratique quotidienne de l'enseignant, l'action («Action»). La phase 2 représente un retour sur l'action, par une description des faits, des émotions vécues, etc. ("Looking back at the action »). La phase 3 est une mise en évidence des aspects essentiels du problème ou de la situation concernée ("Awareness of essential aspects »); la théorie peut être intégrée au processus de réflexion dès ce moment. La phase 4 consiste en une recherche de solutions et en la création de méthodes alternatives ("Creating alternative methods of action»). Enfin, la phase 5, qui comporte une mise à l'essai et un jugement critique des méthodes alternatives ("Trial»), requiert un retour à la pratique pour évaluer les effets des solutions. Le cycle de ce processus de réflexion se poursuit donc de manière continuelle, dans une perspective d'amélioration des compétences et de développement professionnel continu, où l'articulation de la théorie et de la pratique représente une pierre angulaire. L'adaptation que nous avons faite de ce modèle est présentée dans la section Contexte.

\section{Identité professionnelle}

En formation initiale, les différentes identités de la PES sont en interaction. En effet, l'identité personnelle et professionnelle du futur enseignant se développe (Gohier, Anadón, Bouchard, Charbonneau et Chevrier, 2001) en même temps que les savoirs s'articulent entre eux et à la pratique vécue lors des stages. Lorsqu'elle est amenée à expliciter sa pratique, la PES sollicite des arguments qui font tout à la fois référence à son identité personnelle et professionnelle si l'on conçoit, à l'instar de Gohier et al. (2001), que l'identité professionnelle en enseignement renvoie à la représentation 
que l'enseignant a de lui comme personne (rapport à soi) et à celle qu'il a des enseignants et de la profession. La première renvoie à soi, c'est-à-dire aux connaissances, aux valeurs, aux habiletés, aux buts, aux projets ainsi qu'aux aspirations de la personne. La seconde fait intervenir divers rapports reliés au travail, aux responsabilités, aux apprenants, aux collègues, au corps enseignant ou encore à l'école, de manière plus générale. Ces différents rapports sont en dialogue constant, tantôt en continuité, tantôt en rupture les uns avec les autres.

\section{Méthodologie}

Au regard de l'objectif de ce texte qui, rappelons-le, est de décrire les retombées de deux dispositifs pédagogiques de réflexion collective dans le cadre de la formation pratique, cette analyse scientifique à caractère qualitatif s'inscrit dans un paradigme de recherche interprétatif. Une place de première ligne a été donnée à l'acteur lors du recueil des données (Van der Maren, 1996), puisque nous pensons, à l'instar de Savoie-Zajc (2011), que la compréhension de l'action passe par le sens que l'acteur luimême donne au phénomène vécu. C'est pourquoi l'analyse se base sur les commentaires des PES concernées par les dispositifs, collectés lors de l'évaluation des dispositifs proposés à l'université au cours de l'année 2017.

En ce qui concerne le premier dispositif pédagogique, à savoir les cercles pédagogiques, l'évaluation s'est faite auprès des $67 \mathrm{PES}$, à travers une réflexion écrite individuelle à l'issue de chacun des deux cours consacrés aux cercles pédagogiques. Pour un des commentaires, il s'agissait d'identifier les apprentissages réalisés dans le cadre du travail en cercles pédagogiques. Dans l'ensemble, les PES ont rédigé une réflexion d'une longueur d'environ 3 à 5 lignes par commentaire.

En ce qui concerne le second dispositif pédagogique, la démarche d'analyse de cas, l'évaluation s'est faite auprès de $58 \mathrm{PES}$, à travers une réflexion écrite individuelle, à l'issue du cours de séminaire où se déployait le dispositif pédagogique. Dans cette réflexion, les PES devaient entre autres préciser les apprentissages effectués lors de la réalisation de la démarche d'analyse de cas, puis indiquer ce que cette démarche leur apportait de plus que le dispositif des cercles pédagogiques en ce qui concerne le développement de la compétence réflexive.

Les données collectées ont été transcrites intégralement dans Excel, ce qui a permis une analyse systématique par la suite. La procédure choisie pour ce faire est celle de l'analyse thématique de contenu, car les catégories servant à classifier renvoient à des thèmes (Paillé et Mucchielli, 2012). Dans un premier temps, un survol des données a permis de dégager des grands thèmes émergents, qui ont mené à la mobilisation de divers cadres d'analyse. Nous avons donc par la suite adopté deux angles d'analyse distincts au regard des données et selon l'objectif poursuivi. Dans le but de mettre au jour les différents apprentissages des PES dans les cercles pédagogiques, plusieurs thèmes étaient préalablement formulés en lien avec des éléments du cadre conceptuel renvoyant aux différents rapports définissant l'identité professionnelle (Gohier et al., 2001). D'autres thèmes ont aussi émergé des données : l'observation, les questions posées et les rétroactions offertes. Puis, pour les apprentissages des PES lors de la démarche d'analyse de cas, le modèle d'identité professionnelle ne ressortait pas dans les réponses des PES, donc il n'a pas été utilisé pour l'analyse; ici, c'est surtout le processus de réflexion et ses diverses phases (Korthagen et Kessels, 1999; Leroux, 2010) qui ont émergé des données, de même que des 
apprentissages relatifs à la différenciation pédagogique et à l'apport du travail collectif. Dans le cas des deux dispositifs, les autres thèmes émergents représentent des apprentissages qui ne cadraient pas tout à fait dans les modèles retenus (identité professionnelle et ALACT), mais que nous avons néanmoins jugés intéressants. Une fois le codage terminé, une analyse des occurrences pour chacun des codes et des citations pertinentes a été faite pour mener à la présentation de certains résultats.

En ce qui concerne la question sur la complémentarité des deux dispositifs, nous avons d'abord analysé leurs apports respectifs selon les PES. Pour ce faire, nous avons effectué un survol des réponses des PES afin d'identifier des thèmes récurrents, selon une approche émergente. Ceux-ci sont reliés au processus ou à la modalité spécifique du dispositif, aux objets d'analyse qu'il permet d'aborder et, enfin, aux effets attribués à l'un ou à l'autre des dispositifs. Une fois les apports mis en lumière, nous avons jugé intéressant de faire ressortir la complémentarité des deux dispositifs pédagogiques.

De sorte à s'assurer de la validité du codage, un contre-codage des données analysées a été réalisé entre les deux chercheuses. Pour les commentaires des PES au sujet de leurs apprentissages dans le cadre des deux dispositifs, $20 \%$ des commentaires pris au hasard ont été codés indépendamment puis comparés. Le taux d'accord interjuges moyen est de $78 \%$. Pour ce qui est des apports des dispositifs et de leur complémentarité, étant donné le caractère plus émergent du codage, toutes les réponses des PES à cette question ont été codées indépendamment par les deux chercheuses, puis ce codage a été discuté et comparé en vue de l'obtention d'un consensus.

\section{Principaux résultats}

En vue de décrire les retombées des deux dispositifs pédagogiques de réflexion collective, nous nous attardons d'abord aux apprentissages perçus par les PES, puis aux apports respectifs des deux dispositifs et à leur complémentarité.

Apprentissages perçus par les PES

En ce qui concerne les apprentissages perçus par les PES, nous les présentons selon le dispositif de réflexion collective expérimenté, soit les cercles pédagogiques, puis l'analyse de cas.

\section{Cercles pédagogiques}

Le tableau 1 présente les fréquences des codes attribués aux réponses des PES au sujet des apprentissages réalisés à la suite des cercles pédagogiques lors des cours 8 et 9 du laboratoire de $1^{\text {re }}$ année. Il est possible de constater que les aspects relatifs à l'identité sont abordés par presque toutes les PES (92\% et $95 \%)$, surtout en ce qui a trait au :

- rapport aux collègues (et ici aussi à soi) : "Nouvelles perceptions de nous-mêmes. On découvre des habiletés différentes chez nos collègues, dont on ne se doutait pas nécessairement. C'est constructif. » $(\# 18)^{2}$

- rapport au travail : "Il y a différentes méthodes de travail, et les contextes en stage ne sont pas les mêmes pour tous. " (\#6) ou "J'ai appris qu'il y a une panoplie de façons d'aborder une activité et qu'il est bénéfique de varier ses approches d'enseignement. » (\#41) 
- rapport à soi (et ici aussi aux collègues) : "J'ai appris sur ma pratique. J'ai appris à me poser des questions sur ce que je soubaite devenir comme enseignante. Mes collègues mont apporté des points qui ont suscité ma réflexion. " (\#38) ou "J'ai appris à appuyer mes propres actions et à accepter les points de vue des autres. J'ai aussi appris à faire de l'introspection sur ma pratique. » (\#22)

\section{Tableau 1}

Fréquences des codes au sujet des apprentissages réalisés à la suite des cercles pédagogiques.

\begin{tabular}{ccc}
\hline \multicolumn{1}{c}{ Codes } & \multicolumn{2}{c}{ Fréquence } \\
\cline { 2 - 3 } & $\begin{array}{ccc}\text { Cours } \mathbf{8} \\
(\mathbf{N}=\mathbf{6 6})\end{array}$ & $\begin{array}{c}\text { Cours } \mathbf{9} \\
\mathbf{N}=\mathbf{6 3})\end{array}$ \\
\hline Identité & 61 & 60 \\
professionnelle & $92 \%$ & $95 \%$ \\
\hline - rapport collègues & 38 & 38 \\
& $58 \%$ & $60 \%$ \\
\hline - rapport travail & 31 & 39 \\
& $47 \%$ & $62 \%$ \\
\hline - rapport soi & 32 & 32 \\
& $48 \%$ & $51 \%$ \\
\hline - rapport apprenants & 11 & 5 \\
& $16 \%$ & $8 \%$ \\
\hline Observation & 19 & 21 \\
& $29 \%$ & $33 \%$ \\
\hline Rétroactions & 20 & 11 \\
& $30 \%$ & $18 \%$ \\
\hline Questions & 9 & 13 \\
& $14 \%$ & $21 \%$ \\
\hline
\end{tabular}

D’autres codes ont aussi émergé dans les réponses des participants :

- Observation : "J'ai réalisé à quel point me regarder et porter une attention particulière à ma façon d'agir me permet de m'autocritiquer et de m'améliorer. J'ai appris que le point de vue d'une autre personne peut être bénéfique pour moi. » (\#59)

- Rétroactions: "De nombreux apprentissages peuvent être faits grâce aux commentaires des membres de l'équipe. J'ai aimé avoir plusieurs commentaires constructifs qui apportent un autre regard sur ma pratique. »(\#21)

- Questions : "J'ai appris à poser des questions qui ont favorisé l'interaction et à porter une attention particulière sur mes actions. » (\#45)

Considérant ces résultats, nous pouvons constater que les apprentissages perçus des PES concernent davantage les effets de la réflexion, à savoir le développement de certains aspects de l'identité professionnelle, ainsi que de diverses habiletés telles que l'observation et la capacité à offrir des rétroactions. Nous 
sommes conscientes que les codes émergents ne sont peut-être pas de même niveau, mais il nous fallait trouver un moyen d'intégrer les réponses qui ne cadraient pas tout à fait dans le modèle de l'identité professionnelle ou, à tout le moins, qui allaient plus loin.

\section{Analyse de cas}

Le tableau 2 présente les fréquences des codes attribués aux réponses des PES concernant les apprentissages réalisés grâce à la démarche d'analyse de cas lors du séminaire de $2^{\mathrm{e}}$ année.

\section{Tableau 2}

Fréquences des codes concernant les apprentissages réalisés grâce à la démarche d'analyse de cas.

\begin{tabular}{|c|c|}
\hline Codes & $\begin{array}{l}\text { Fréquence } \\
(\mathrm{N}=58)\end{array}$ \\
\hline Processus & $\begin{array}{c}51 \\
88 \%\end{array}$ \\
\hline - $\quad$ Phase 1 (action) & $\begin{array}{c}3 \\
5 \%\end{array}$ \\
\hline - $\quad$ Phase 2 (retour sur l'action - analyse des faits et besoins) & $\begin{array}{c}20 \\
35 \%\end{array}$ \\
\hline - $\quad$ Phase 3 (problème) & $\begin{array}{c}29 \\
50 \%\end{array}$ \\
\hline - $\quad$ Phase 4 (solutions) & $\begin{array}{c}26 \\
45 \%\end{array}$ \\
\hline - $\quad$ Phase 5 (avantages et limites des solutions) & $\begin{array}{c}13 \\
22 \%\end{array}$ \\
\hline Différenciation pédagogique (DP) & $\begin{array}{c}10 \\
17 \% \\
\end{array}$ \\
\hline Apport du collectif & $\begin{array}{c}7 \\
12 \%\end{array}$ \\
\hline
\end{tabular}

On peut ainsi remarquer que la plupart des PES (88 \%) reviennent surtout sur le processus de réflexion et non sur l'identité professionnelle, tel que mentionné plus tôt :

- "Les différentes étapes à suivre pour faire une analyse de cas. Phase 1 à 5 . Il est important de prévoir plusieurs solutions. » (\#29)

- "J'ai appris à ne pas me diriger vers les solutions en premier lieu. Il faut d'abord que j’analyse la situation dans sa globalité. Ainsi, je m’appuierai sur les faits dorénavant, plutôt que de juger la situation trop rapidement. » (\#54)

Plusieurs mettent un accent particulier sur certaines phases du processus :

- 3 (problème) : "J'ai appris quill faut bien cibler un problème par les faits pour trouver des bonnes solutions. De plus, j'ai compris que c'est très subjectif de trouver un problème, car nous avons tous perçu les faits de différentes façons. » (\#47) 
- 4 (solutions)... et 5 (jugement) : - "J'ai appris que dans chaque solution, il y aura des avantages et des limites. C'est à nous de les trouver. Il faut bien clarifier les besoins des élèves pour bien les aider. " (\#10)

Aussi, il est à noter que des codes émergents permettent d'élargir le spectre des apprentissages au-delà du processus lui-même, notamment en lien avec :

- la différenciation pédagogique : "Cette démarche m’a permis d'analyser ma propre pratique en ciblant certains aspects de la planification qui doivent être considérés avant le pilotage de nos activités d'enseignement; l'importance de bien prévoir et anticiper les besoins des élèves en fonction de leurs caractéristiques et manières d'apprendre; l'importance aussi de préciser son intention pédagogique et d'orienter des stratégies d'enseignement en fonction de l'atteinte de celles-ci. Prévoir! Prévoir! Prévoir! Orienter notre planification en fonction d'une différenciation. » (\#58)

- l'apport du collectif : "J'ai appris que certaines choses que l'on vit peuvent et sont souvent vécues par d'autres que nous. De plus, ne pas avoir peur d'en parler, car il y a des pistes de solutions qui peuvent être amenées par les autres qui peuvent être très bénéfiques. » (\#36)

Ainsi, en ce qui concerne les apprentissages perçus par les PES à l'issue de l'analyse de cas, c'est plus le processus de réflexion lui-même qui est évoqué, particulièrement les phases 2 à 4 .

\section{Apports et complémentarité des dispositifs}

De nombreux apports, tant à l'égard de la démarche d'analyse de cas que des cercles pédagogiques, ont été mentionnés par les PES lorsqu'elles ont été amenées à indiquer ce que cette démarche leur apportait de plus que le dispositif des cercles pédagogiques du point de vue de la compétence réflexive. Ces apports ont été regroupés selon trois catégories principales : le processus (ou la modalité) spécifiquement relié au dispositif; les objets d'analyse que le dispositif permet d'aborder; et les effets qui en découlent. Ainsi, les différents apports seront présentés selon ces trois catégories pour chacun des deux dispositifs analysés.

Le tableau 3 présente les fréquences d'apparition des codes attribués aux réponses des PES (collectées à l'issue du séminaire de $2^{\mathrm{e}}$ année) au sujet des apports des deux dispositifs.

\section{Tableau 3}

Fréquences d'apparition des codes au sujet des apports des deux dispositifs.

\begin{tabular}{lcc}
\hline \multirow{2}{*}{ Codes } & \multicolumn{2}{c}{ Fréquences } \\
\cline { 2 - 3 } & Analyse de cas & Cercles pédagogiques \\
\hline Processus (ou modalité) & 43 & 12 \\
& $\mathbf{8 6 \%}$ & $\mathbf{5 2 \%}$ \\
\hline Objets d'analyse & 35 & 16 \\
& $\mathbf{7 0 \%}$ & $\mathbf{7 0} \%$ \\
\hline Effets & 22 & 7 \\
& $\mathbf{4 4 \%}$ & $\mathbf{3 0} \%$ \\
\hline Total & $\mathbf{5 0 3}$ & $\mathbf{2 3}$ \\
\hline
\end{tabular}




\section{Cercles pédagogiques}

On peut remarquer que plus des deux tiers des commentaires $(69,5 \%)$ des PES concernent les objets d'analyse. Les PES mentionnent que les cercles pédagogiques portent sur leurs expériences et leur propre pratique, à travers leur justification ou leur évaluation (tel qu'il est mentionné plus haut). Les objets d'analyse sont spécifiques aux PES ou à leur contexte de stage :

- "Les cercles pédagogiques étaient plus spécifiques à nous et à notre leçon. »

En cela, les PES soulignent le caractère réel de l'objet sur lequel le dispositif permet de porter son attention. Certaines PES expriment même une préférence pour les cercles pédagogiques pour son caractère personnalisé :

- «...j’ai préféré regarder les vidéos, car cela est plus personnel et ça parle de sujet vécu. » (\#38)

Plus de la moitié des commentaires (52\%) des PES à l'égard des cercles pédagogiques ont trait au processus. Ces dernières mentionnent que le dispositif permet de faire une introspection et de porter un regard sur soi plus spécifiquement. Elles le voient comme un processus de justification de la pratique et d'évaluation de l'expérience :

- "Cette démarche [l'analyse de cas] permet de trouver des solutions concrètes, alors que les cercles pédagogiques nous amenaient à justifier notre pratique. » (\#17)

Également, le processus à l'œuvre dans les cercles pédagogiques permet d'évaluer ses pairs et favorise l'émergence d'idées en vue de se développer sur le plan professionnel :

- "Les cercles pédagogiques étaient bien pour évaluer les autres et trouver des idées pour nous améliorer. » (\#35)

À peu près le tiers des commentaires (30\%) des PES concernent les effets du dispositif. Les PES notent que les cercles pédagogiques offrent de développer leur compétence réflexive sur leur démarche enseignante, en plus de leur permettre d'améliorer leur enseignement :

- «[...] les cercles pédagogiques nous ont permis de nous améliorer dans notre façon d'enseigner. » (\#56)

\section{Analyse de cas}

Une large part des commentaires (86\%) des PES concernent le processus (ou la modalité) spécifiquement relié au dispositif. L'analyse de cas est appréciée, car elle permet particulièrement de bénéficier des arguments, des idées et des commentaires de plusieurs personnes. Les PES notent qu'elle les amène, par le biais de l'entraide et l'échange, à réfléchir d'une façon différente. Le processus propre au dispositif semble aussi soutenir l'organisation de la réflexion :

- "Les étapes de la démarche réflexive permettent de mieux organiser nos idées et elles permettent de nous guider dans l'élaboration de pistes de solution. » (\#15)

Cette idée de structuration de la pensée revient plusieurs fois et les PES soulignent les étapes précises que propose ce modèle qu'elles qualifient même de «concret» et "complet ", ce qui leur évite de « dévier » ou de passer trop rapidement aux solutions avant d'approfondir l'analyse du cas :

- «Nous sommes guidés et structurés, ce qui permet d’avancer selon des étapes précises. » (\#10) 
- "On a tendance à sauter à des solutions trop rapidement sans avoir analysé le problème en profondeur. " (\#53)

L'analyse de cas offre une certaine distance sur les problèmes traités et reliés au pilotage, car le cas faisant l'objet d'analyse ne concerne pas directement les PES :

- "Elle nous permet d'avoir un regard plus distant face à une problématique et à faire davantage d'objectivation de notre pratique enseignante. Nous avons parfois plus de facilité à analyser une situation problématique vécue par quelqu'un d'autre que la nôtre (plus subjectif-émotions). Les solutions sont plus faciles à cibler. » (\#60)

Environ deux tiers des commentaires des PES portent sur les objets d'analyse (70 \%). L'analyse de cas cible des situations particulières vécues en contexte réel et offre de réfléchir à des situations tierces, ce que les PES jugent pertinent pour le futur travail. Cette démarche permet d'analyser leur pratique sous l'angle de l'intervention, comme les techniques d'enseignement. Le regard porté ne se restreint pas à « soi », mais semble s'ouvrir aux élèves et à l'ensemble du groupe :

- « Elle [la démarche réflexive] nous amène à réfléchir non seulement à nous, mais aux élèves et aux conséquences de notre façon d'enseigner. » (\#41)

En plus de porter sur des situations problématiques qui demandent à être traitées, la réflexion suscitée par cette démarche porte sur la manière de résoudre un problème :

- "Cette pratique nous permet de cibler les problèmes en particulier, tout en s'intéressant à la façon dont nous allons s'y prendre pour les régler. » (\#50)

Une bonne part de commentaires (44\%) des PES portent sur les effets du dispositif. Lorsque les PES sont amenées à comparer les cercles pédagogiques à l'analyse de cas, elles estiment que cette dernière suscite davantage le questionnement et la réflexion :

- "Je trouve que cette démarche suscite davantage la réflexion et le questionnement. Elle permet une meilleure prise de conscience des besoins des élèves et des interventions à privilégier. Cela permet de mieux organiser mes idées. » (\#12)

En plus de permettre d'anticiper l'action, d'organiser ses idées pour mieux comprendre sa pratique, l'analyse de cas permet aussi d'adapter cette dernière en tenant compte des besoins des élèves pour différencier ses interventions :

- "Elle nous amène à réfléchir sur d'éventuels cas qui pourraient arriver et à nous préparer à l'avance." (\#7)

- "Cette démarche réflexive m’apporte des outils pour adapter ma pratique aux besoins des élèves. " (\#30)

- "Cette démarche nous permet de différencier nos interventions et d'avoir des pistes sur la manière d'intervenir lors des cas plus complexes. » (\#43)

L'extrait qui suit résume de quelle façon la démarche réflexive peut contribuer à la formation des PES à la fois en termes de processus, d'objets de réflexion et d'effets :

- " Cette démarche est très concrète, elle nous amène à réfléchir constamment. Elle est très complète et nous permet de trouver des pistes de solutions pour le problème. Je trouve la démarche d'analyse très intéressante, rapide et utile. L'activité d'aujourd'bui me permet de réfléchir sur la façon dont je réagirais face à une situation.» (\#24) 
Par ailleurs, les deux dispositifs sont vécus positivement et les PES cernent leur complémentarité. Les extraits qui suivent en témoignent, tout en mettant de l'avant leur utilité :

- "Je trouve que les deux activités amènent leurs points forts de manière différente et quelles sont utiles tout autant. D'ailleurs, c'est agréable de vivore différentes activités. " (\#6)

- "La démarche réflexive aidera les cercles pédagogiques en organisant l’information sur une problématique quelconque.» (\#53)

\section{Discussion}

Globalement, ces résultats permettent de bien cerner les retombées, les apprentissages, de même que les apports respectifs et complémentaires des dispositifs étudiés.

L'analyse des apprentissages perçus par les PES révèle que les cercles pédagogiques ont permis de cibler plusieurs dimensions identitaires chez eux, particulièrement le rapport aux collègues, au travail et à soi (Gohier et al., 2001) et offrent ainsi d'aller toucher le "pourquoi » de l'action et non seulement l'action elle-même, comme valorisé par Haugan, Moen et Karlsdottir (2013). De plus, les cercles pédagogiques ont révélé le potentiel de l'observation par la vidéo (de soi et des autres) pour le développement professionnel des PES (Meschede, Fiebranz, Möller et Steffensky, 2017; O'Leary, 2014; Wragg, 2012), de même que l'importance de la réflexion collective (Collier, 2010; Correa Molina et al., 2010; Parkison, 2009). En effet, les questions et les rétroactions des collègues ont agi comme moteur de réflexion pour les $\mathrm{PES}$, de même que leurs propres questions et rétroactions adressées aux collègues, ce que nous avons appelé l'effet «miroir » (je [A] pose une question à Autrui [B] et elle me revient ensuite, car je m'interroge en même temps sur cet aspect (A double flèche B) (Leroux, Laflamme et Vivegnis, 2017). Il est possible de voir ici émerger l'idée de " réflexion partagée » (Tochon, 1996, cité dans Malo et Desrosiers, 2011). Ce sont donc ici des effets de la réflexion qui ressortent davantage en termes de retombées.

Pour ce qui est des apprentissages reliés à l'analyse de cas, ils concernent principalement le processus d'apprentissage, surtout pour la phase d'identification du problème, avant d'aller vers la recherche de pistes de solutions. Par ailleurs, il est intéressant de noter que certaines PES ont aussi relevé des apprentissages relatifs à la différenciation pédagogique, de même qu'à l'apport du collectif dans cette démarche (Collier, 2010), notamment en ce qu'il permet l'expression de perspectives et de points de vue multiples (Correa Molina et al., 2010), ce qui renforce notre choix de dispositifs de réflexion collective. Conséquemment, les principales retombées de ce dispositif concernent le processus de réflexion.

Enfin, la complémentarité des dispositifs a réellement été mise en exergue grâce à l'analyse de leurs apports respectifs, en permettant un travail à la fois sur le processus, les objets d'analyse et les effets de la réflexion. Alors que les cercles pédagogiques offrent, à travers un processus particulier, de porter un regard sur soi, que ce soit par la justification ou l'évaluation de l'expérience, l'analyse de cas offre avant tout d'organiser sa pensée et d'aiguiser ses arguments en vue de résoudre un problème. Les deux dispositifs offrent une prise de recul sur la situation, nécessaire à l'apprentissage en stage (Donnay, 2008), l'un de façon plus personnalisée (les cercles), l'autre avec davantage de distance (l'analyse de cas), en fonction du cas à l'étude. 
En termes d'objets d'analyse, si les cercles pédagogiques permettent de focaliser l'attention sur des expériences vécues par les PES elles-mêmes et les fondements sous-jacents à l'action (Vesterinen et al., 2014), l'analyse de cas se concentre sur des situations tierces avec un regard porté également sur les élèves et le groupe. La focale est donc élargie, mettant en exergue des préoccupations variées, telles qu'explorées dans divers modèles de réflexion (Conway et Clark, 2003; Haugan et al., 2013; Korthagen et Vasalos, 2005), mais dans les deux cas, le caractère réel et concret des objets d'analyse est souligné (Chaubet, 2010).

Quant aux effets de la réflexion, les cercles pédagogiques offrent avant tout de développer la compétence réflexive en lien avec la démarche enseignante, permettant selon les PES d'améliorer leur enseignement. En ce qui concerne l'analyse de cas, les effets ciblent davantage l'apprentissage d'une démarche de réflexion permettant de mieux anticiper l'action et son adaptation aux élèves. La démarche systématique qu'offre l'analyse de cas permet une conscientisation approfondie du problème traité. Par les rétroactions et les questions des pairs, les cercles soutiennent, pour leur part, les PES en les outillant concrètement pour leur propre pratique. Et si davantage de commentaires portent sur les effets à l'égard du dispositif d'analyse de cas (44\%), cela s'expliquerait en partie par le fait que ceux-ci sont très étroitement liés au processus. Par exemple, l'analyse de cas permet de trouver des solutions, ce qui met de l'avant le processus en même temps que son effet.

\section{Conclusion}

Certaines limites caractérisent l'étude dont il est ici question. Au départ, nous n'avions pas d'intention de recherche. Les questions adressées aux PES dans le cadre des cours de laboratoire et de séminaire ont été posées dans un esprit d'objectivation de l'enseignement. Puis, au regard des réponses obtenues, nous avons jugé qu'il s'agissait de données intéressantes à analyser pour évaluer les apports respectifs des dispositifs exploités. Il faut aussi noter que lorsque nous avons questionné les PES au sujet de la complémentarité des dispositifs au moment du séminaire, des effets de décalage dans le temps (environ 6 mois entre les deux dispositifs) ont pu influencer les réponses, car l'analyse de cas a eu lieu plus d'un trimestre après les cercles pédagogiques. Selon Correa Molina et al. (2010), la distance temporelle relative à un événement influencerait la qualité de la réflexion. De plus, il se peut que la documentation des apports ait été influencée par formulation de la question posée aux PES à l'issue du séminaire ayant permis d'aborder l'analyse de cas (Indiquez ce que cette démarche vous a apporté de plus que le dispositif des cercles pédagogiques...).

En dépit de ces limites, les résultats permettent par ailleurs de dégager des pistes pour la formation initiale. Les deux dispositifs que nous avons explorés s'avèrent à notre avis complémentaires et essentiels pour le développement de l'identité professionnelle et pour amener les PES à apprendre à réfléchir de manière autonome (Svojanovsky, 2017). L'analyse de cas offre de travailler sur une démarche de réflexion plus transversale, centrée sur la résolution de problèmes pratiques, mais décentrée des PES (pratique des autres pairs, mais aussi regard sur l'apprentissage des élèves), ce qui permet d'une certaine façon d'élever les considérations au-delà des particularités subjectives et des contextes d'enseignement individuels (Fazio, 2009). Elle porte ainsi une visée à plus long terme pour le développement des PES, en permettant de passer de préoccupations centrées sur soi à d'autres davantage reliées à la qualité de l'enseignement offert et à son impact sur les élèves (Fuller et Bown, 1975; Korthagen et Wubbels, 2001; Ong'ondo et Jwan, 2009). Les cercles pédagogiques s'appuyant sur la vidéoscopie permettent un travail 
plus spécifique pour soi et sont donc plus significatifs pour certains. Ils sont basés sur l'introspection de la PES, l'analyse de sa pratique et de son vécu, ce qui constitue des activités " réelles " de travail, plus porteuses de réflexion selon Chaubet (2010). Ils sont centrés sur la justification des fondements de sa pratique, un aspect moins observable, ce qui contribue à la prise de conscience de la PES et au développement de son identité professionnelle. Toutefois, jugés plus complexes sur le plan technique, les cercles pédagogiques demandent à ce que les PES soient accompagnées lors de leur utilisation. Dans ce sens, la complémentarité des dispositifs permet d'envisager la réflexion dans une perspective développementale, en offrant de complexifier le processus par une diversification des ressources, des objets et des modèles de réflexion (Correa Molina et al., 2010).

En outre, cette mise en œuvre de deux dispositifs de réflexion collective permet de dégager certains enjeux pour la formation des PES. D'abord, nous avons pu constater que lors des cercles pédagogiques, plusieurs PES donnaient des rétroactions à leurs pairs, plutôt que de leur poser des questions pour expliciter leur pratique. Cela amenait les pairs en question à se défendre au lieu de justifier leur choix et d'argumenter sur les fondements de leur agir. Comme formatrices et formateurs, nous aurions dès lors à travailler au développement de l'habileté des PES à questionner plutôt que de se juger mutuellement. Aussi, bien que nous ayons constaté les nombreux apports de la dimension collective dans les dispositifs de réflexion proposés, cet aspect comporte certains défis. Lors des cercles pédagogiques, des PES ont dit :

- «Difficile de seulement poser des questions sans commenter avec nos opinions (critiques). " (\#77)

- "Difficile de ne pas poser des questions de nature critique. " (\#78)

Pour ce qui est de l'analyse de cas, une PES précise que c'est :

« difficile étant donné que les opinions peuvent différer. Il peut alors être difficile de s'entendre. » (\#20)

Ce sont ici les habiletés à délibérer, à exprimer ses opinions, puis à trouver des compromis satisfaisants pour tous qui sont mises de l'avant. Ainsi, ces dispositifs peuvent réellement constituer des leviers de développement pour les PES, pour autant que les formatrices et formateurs les soutiennent dans le développement d'habiletés sous-jacentes, qui rendent leur mise en œuvre possible et bénéfique.

Pour terminer, les résultats présentés ici, bien que préliminaires et exploratoires, permettent de dégager le fort potentiel des dispositifs employés. D'autres études plus poussées et menées de manière systématique pourraient permettre de confirmer les apports de ceux-ci pour la formation des futurs enseignants.

\section{Notes}

1 Dans notre institution, lorsqu'il est question d'évaluation d'activités pédagogiques, sans recueillir de données confidentielles des participants, seule une déclaration éthique est nécessaire.

2 Les PES sont identifiées par des numéros (\#) renvoyant à l'ordre d'apparition de leurs commentaires dans le fichier Excel utilisé lors du traitement des données. Ici, il est question de la PES dont le commentaire apparait à la ligne 18 du document.

350 représente le nombre total de fois où les PES ont fait mention de l'analyse de cas dans leurs commentaires.

423 représente le nombre total de fois où les PES ont fait mention des cercles pédagogiques dans leurs commentaires. 


\section{Références}

Chaubet, P. (2010). Saisir la réflexion pour mieux former à une pratique réflexive : d'un modèle théorique à son opérationnalisation. Éducation et francophonie, 38(2), 60-77. http://dx.doi.org/10.7202/1002164ar

Collier, S. T. (2010). Reflection as a social problem-solving process. Dans E. G. Pultorak (dir.), The purposes, practices, and professionalism of teacher reflectivity: Insights for twenty-first-century teachers and students (p. 45-71). Lanham, MD : Rowman and Littlefield Education.

Conway, P. F. et Clark, C. M. (2003). The journey inward and outward: a re-examination of Fuller's concerns-based model of teacher development. Teaching and Teacher Education, 19(5), 465-482. http://dx.doi.org/10.1016/s0742-051x(03)00046-5

Correa Molina, E., Collin, S., Chaubet, P. et Gervais, C. (2010). Concept de réflexion : un regard critique. Éducation et francophonie, 38(2), 135-154. http://dx.doi.org/10.7202/1002160ar

Correa Molina, E. et Gervais, C. (2010). Une adaptation de l'approche de Fenstermacher pour explorer la communication du savoir d'expérience. Dans F. Yvon et F. Saussez (dir.), Analyser l'activité enseignante : des outils méthodologiques et théoriques pour l'intervention et la formation (p. 229-250). Québec, QC : Presses de l'Université Laval.

Donnay, J. (2008). Préface. Dans M. Boutet et J. Pharand (dir.), L'accompagnement concerté des stagiaires en enseignement (p. XI-XIII). Québec, QC : Presses de l'Université du Québec.

Fazio, X. (2009). Teacher development using group discussion and reflection. Reflective Practice, 10(4), 529-541. http://dx.doi.org/10.1080/14623940903138407

Fenstermacher, G. D. (1987a). A reply to my critics. Educational theory, 37(4), 413-422. http://dx.doi.org/10.1111/j.1741-5446.1987.00413.x

Fenstermacher, G. D. (1987b). Prologue to my critics. Educational theory, 37(4), 357-360. http://dx.doi.org/10.1111/j.1741-5446.1987.00357.x

Fuller, F. F. et Bown, O. H. (1975). Becoming a teacher. Dans K. Ryan (dir.), Teacher education (74 ${ }^{\text {th }}$ Yearbook of the National Society for the Study of Education) (part 2, p. 25-52). Chicago, IL : University of Chicago Press.

Gohier, C., Anadón, A., Bouchard, Y., Charbonneau, B. et Chevrier, J. (2001). La construction identitaire de l'enseignant sur le plan professionnel : un processus dynamique et interactif. Revue des sciences de l'éducation, 27(1), 3-32. http://dx.doi.org/10.7202/000304ar

Haugan, J. A., Moen, T. et Karlsdottir, R. (2013). Exploration of Norwegian student teachers' reflective mediation during internships. Reflective Practice, 14(2), 226-239. http://dx.doi.org/10.1080/14623943.2012.749233

Korthagen, F. A. J. et Kessels, J. P. A. M. (1999). Linking theory and practice: Changing the pedagogy of teacher education. Educational Researcher, 28(4), 4-17. http://dx.doi.org/10.3102/0013189x028004004

Korthagen, F. A. J. et Vasalos, A. (2005). Levels in reflection: Core reflection as a means to enhance professionnal growth. Teachers and Teaching: Theory and Practice, 11(1), 47-71. http://dx.doi.org/10.1080/1354060042000337093

Korthagen, F. A. J. et Wubbels, T. (2001). Characteristics of reflective teachers. Dans F. A. J. Korthagen, J. Kessels, B. Lagerwerf et T. Wubbels (dir.), Linking practice and theory: The pedagogy of realistic teacher education (p. 131-148). Mahwah, NJ : Lawrence Erlbaum Associates.

Leroux, M. (2010). Étude des relations entre la résilience d'enseignantes et d'enseignants du primaire cuvrant en milieux défavorisés et la réflexion sur la pratique (Thèse de doctorat, Université de Montréal). Repéré à https://papyrus.bib.umontreal.ca/xmlui/handle/1866/3946

Leroux, M., Laflamme, D. et Vivegnis, I. (2017, juillet). Mise en ceuvre d'un dispositif d'alternance en formation à l'enseignement : entre finalités et vécu subjectif des apprenants. Communication présentée aux XV rencontres internationales du Réseau de recherche en éducation et en formation (RÉF) - Symposium 10 : L'analyse et la compréhension des points aveugles dans l'alternance en éducation et en formation, Paris, France.

Malo, A. et Desrosiers, P. (2011). Un dispositif visant à soutenir la pratique réflexive en stage : démarche de mise en mots et dynamique des échanges entre enseignants et stagiaires au sujet de situations de pratique. Dans F. Guillemette et M. L'Hostie (dir.), Favoriser la progression des stagiaires en enseignement (p. 91-120). Québec, QC : Presses de l'Université du Québec. 
Meschede, N., Fiebranz, A., Möller, K. et Steffensky, M. (2017). Teachers' professional vision, pedagogical content knowledge and beliefs: On its relation and differences between pre-service and in-service teachers. Teaching and Teacher Education, 66, 158-170. http://dx.doi.org/10.1016/j.tate.2017.04.010

Ministère de l'Éducation du Québec (MEQ). (2001). La formation en enseignement. Les orientations. Les compétences professionnelles. Repéré à http://www.education.gouv.qc.ca/fileadmin/site web/documents/reseau/formation titularisation/formation enseignement orientations EN.pdf

O'Leary, M. (2014). Classroom observation: A guide to the effective observation of teaching and learning. New York, NY : Routledge.

Ong'ondo, C. O. et Jwan, J. O. (2009). Research on student teacher learning, collaboration and supervision during the practicum: A litterature review. Educational Research and Review 4(11), 515-524. Repéré à http://www.academicjournals.org/app/webroot/article/article1379617229 Ong'ondo\%20and\%20Jwan.pdf

Paillé, P. et Mucchielli, A. (2012). L'analyse qualitative en sciences humaines et sociales. Paris : Armand Colin. http://dx.doi.org/10.3917/arco.paill.2012.01

Parkison, P. T. (2009). Field-based preservice teacher research: Facilitating reflective professional practice. Teaching and Teacher Education, 25(6), 798-804. http://dx.doi.org/10.1016/j.tate.2008.11.017

Portelance, L. et Durant, N. (2006). La collaboration au sein d'une équipe pédagogique, une compétence à développer au cours des stages. Journal of the Canadian Association for Curriculum Studies, 4(2), 77-99. Repéré à https://jcacs.journals.yorku.ca/index.php/jcacs/article/viewFile/17006/15808

Savoie-Zajc, L. (2011). La recherche qualitative / interprétative en éducation. Dans T. Karsenti et L. Savoie-Zajc (dir.), La recherche en éducation : étapes et approches (3e éd., p. 123-147). St-Laurent, QC : ERPI.

Svojanovsky, P. (2017). Supporting student teachers' reflection as a paradigm shift process. Teaching and Teacher Education, 66, 338-348. http://dx.doi.org/10.1016/j.tate.2017.05.001

Thibodeau, S., Dussault, M., Frenette, É. et Royer, N. (2011). Solitude professionnelle d'enseignants du secondaire : relations avec le leadership du directeur d'école et leurs croyances d'autoefficacité sociale. Revue canadienne de l'éducation, 34(4), 177-199. Repéré à http://journals.sfu.ca/cje/index.php/cje-rce/article/view/1081

Van der Maren, J.-M. (1996). Méthodes de recherche pour l'éducation (2éd.). Bruxelles : De Boeck.

Vesterinen, O., Toom, A. et Krokfors, L. (2014). From action to understanding - student teachers' learning and practical reasoning during teaching practice. Reflective Practice, 15(5), 618-633. http://dx.doi.org/10.1080/14623943.2014.900028

Wragg, E. C. (2012). An introduction to classroom observation (2 éd.). New York, NY : Routledge.

\section{Pour citer cet article}

Leroux, M. et Vivegnis, I. (2019). Cercle pédagogique et analyse de cas : dispositifs complémentaires de réflexion collective au coeur de la formation initiale des enseignants. Formation et profession. 27(2), 58-73. $\underline{\text { http://dx.doi.org/10.18162/fp.2019.506 }}$ 